AUTORES:

Sílvio Ramadas ${ }^{1}$

Sidónio Serpa ${ }^{1}$

António Rosado ${ }^{1}$

${ }^{1}$ Faculdade de Motricidade Humana

Universidade Técnica de Lisboa, Portugal

https://doi.org/10.5628/rpcd.12.02.28

\section{Relação entre características psicológicas e nível de desempenho em jovens jogadores de futebol.}

PALAVRAS CHAVE:

Talento. Futebol. Características psicológicas.

\title{
RESUMO
}

0 objetivo do presente estudo foi examinar a relação entre várias características psicológicas e o nível de desempenho em jovens jogadores de futebol no âmbito de um clube de futebol de elite. Setenta e dois jogadores dispensados com idades compreendidas entre os treze e os dezoito anos $(M=15.93$; $D P=.16)$ e sessenta e três jogadores retidos com idades compreendidas entre os catorze e os dezoito anos $(M=15.26$; $D P=.15)$ participaram neste estudo. Foram avaliados os seguintes construtos: compromisso, motivação, resiliência, coping, suporte parental e perfecionismo. Realizou-se uma análise de covariância multivariada, mantendo a variável idade como covariante. De acordo com os resultados, os jogadores retidos revelaram um perfil psicológico mais ajustado às exigências do desporto, obtendo pontuações mais elevadas nas dimensões competência pessoal, motivação intrínseca (conhecimento e estimulação) e secundarização da escola, e pontuações mais baixas ao nível das dúvidas na acção, criticismo parental, motivação extrínseca (identificada e externa), amotivação e rejeição da mãe. Estes resultados corroboram pesquisas anteriores que avaliaram os fatores psicossociais associados ao sucesso de jovens jogadores de futebol de elite. 
Relationship between

psychological characteristics

and level of performance

in young soccer players.

\section{ABSTRACT}

This purpose of the present study was to examine the relationship among several psychological characteristics and the level of performance considering a sample of a youth Portuguese elite football players. Seventy two players aged between thirteen and eighteen $(M=15.93$; $S D=.16)$ released from their club and sixty three selected players aged between fourteen and eighteen $(M=15.26 ; S D=.15)$ retained in their club were involved in this research. The following constructs were evaluated: commitment, motivation, resilience, coping, parental support and perfectionism. A multivariate analysis of covariance was used with age as covariate. According to the results, retained players revealed a more adjusted psychological profile, scoring higher on personnel competence, intrinsic motivation (to knowledge and stimulation) and school subordination, and lower on doubts about action, parental criticism, identified regulation, external regulation, amotivation and mother rejection. Current results reinforce previous studies that focus on the psychosocial factors associated with youth elite soccer success.

\section{KEY WORDS:}

Talent. Football. Psychological characteristics. 


\section{INTRODUÇÃo}

A compreensão dos fatores que diferenciam normalidade e excecionalidade, em particular os catalisadores intrapessoais e ambientais ${ }^{(16)}$ do processo de desenvolvimento do talento, têm constituído um objetivo desafiador para a comunidade científica. As pesquisas realizadas no âmbito da psicologia do desporto, nomeadamente as que se referem ao talento e à carreira dos atletas de elite, têm-se caracterizado por uma elevada complexidade em consequência da natureza multidimensional e dinâmica desta temática ${ }^{(53)}$. Consequentemente as abordagens metodológicas atuais tendem a seguir modelos não lineares de desenvolvimento ${ }^{(44)}$, centrando a atenção em perspectivas multidimensionais e que consideram o trajeto de vida dos atletas, os diferentes papéis que assumem na sociedade, assim como os contextos específicos em que aprendem ${ }^{(55)}$. Esta abordagem, não tradicional e holística, deriva de uma mudança epistemológica e metodológica neste domínio (11) que reforça a orientação dos modelos para o conceito de desenvolvimento em detrimento do conceito de identificação de talento. No entanto, esta conceção nem sempre é sustentada em termos operacionais, uma vez que no terreno os termos identificação e seleção continuam a ter implicações excessivamente relevantes.

As distintas etapas de desenvolvimento da carreira dos atletas requerem tomadas de decisão específicas. Assim, todos os intervenientes neste processo, em particular os próprios praticantes, deverão ter em conta que, no sistema desportivo atual, a partir da etapa de especialização apenas uma quantidade limitada de jogadores integrará uma equipa. Por outro lado, é possível que em cada semana um número reduzido destes jogadores seja convocado para a competição e, destes, apenas alguns irão jogar. Nesta lógica, é pois indiscutível que o termo seleção faz parte das rotinas diárias dos treinadores. Em particular no que diz respeito à formação desportiva de jovens, estas opções podem constituir verdadeiros dilemas para os treinadores que são solicitados a tomar decisões de curto prazo frequentemente com consequências irreversíveis a longo prazo. A vertente mais preocupante deste processo é que, pelo menos com base no conhecimento científico contemporâneo, as dúvidas sobre a objectividade dos procedimentos de identificação parecem persistir. Efetivamente, apesar da capacidade de reconhecer a excecionalidade ser acessível para uma parte considerável dos treinadores que referem que conseguem "ver" o talento ${ }^{(28)}$, os critérios que suportam estas decisões são difíceis de interpretar no plano científico. Adicionalmente, os procedimentos de seleção com base em indicadores fisiológicos e psicológicos parecem não constituir uma alternativa credível a esta forma subjetiva de avaliação da performance (63).

Para além da escassez de critérios científicos neste domínio, o fenómeno de seleção de talentos, frequentemente valorizado no sistema desportivo, parece ter consequências perniciosas. De facto, a disponibilidade de oportunidades de desenvolvimento alicerçado no sucesso a curto prazo parece orientar os treinadores a selecionar os jogadores que, no momento, demonstram melhores prestações em detrimento do potencial a longo prazo. As 
evidências sugerem que estas opções são quase impossíveis de inverter ${ }^{(37)}$. Na realidade, ao longo das últimas décadas, constata-se uma diversidade de pesquisas que corroboram a relação entre o processo de seleção no futebol e o "efeito relativo da idade" $(28,33,52,60)$. De acordo com esta constatação, os procedimentos de seleção no futebol tendem a discriminar favoravelmente os jogadores que nasceram mais cedo no respetivo grupo de idade. Os fundamentos para esta incidência baseiam-se no facto destes jogadores, comparativamente com os seus pares, serem por norma mais experientes e fisicamente mais desenvolvidos ${ }^{(29)}$. As opções com base nestes pressupostos poderão ter implicações indesejáveis a longo prazo pois impedem que muitos jovens possam beneficiar dos mesmos recursos humanos e materiais. 0 "desperdício de talento", na sequência da operacionalização de critérios enviesados, consubstanciam, nesta ótica, um risco a ponderar.

Em alternativa é sugerido que os processos de identificação sejam menos valorizados e que maior atenção seja dedicada aos procedimentos centrados no conceito de desenvolvimento. Neste sentido, um processo de avaliação contínuo e multidimensional deverá ser implementado reconhecendo a relevância dos processos interativos e compensatórios ${ }^{(1)}$. Tal como mencionado anteriormente, parece óbvio que os procedimentos de seleção são parte integrante do processo de treino. No entanto, a capacidade de evolução e o comportamento dos jogadores devem igualmente ser considerados durante os programas de desenvolvimento ${ }^{(1)}$. Torna-se desta forma capital compreender os aspetos psicossociais e ambientais numa perspetiva longitudinal focando a atenção quer nos mais bem sucedidos, quer nos menos bem sucedidos ${ }^{(38)}$. Esta conceção tem implicações relevantes para a psicologia do desporto, uma vez que várias capacidades podem ser fomentadas. As evidências suportam a manifestação de características psicossociais cruciais por parte dos atletas de elite tais como o perfecionismo ajustado, a eficiência na utilização de recursos de coping ${ }^{(23)}$, a resiliência ${ }^{(20)}$, a autoregulação ${ }^{(32)}$, ou o compromisso ${ }^{(45,46)}$. Neste domínio, podemos também acrescer a relevância de algumas variáveis sociodemográficas e ambientais no âmbito do processo de desenvolvimento do talento que têm sido identificadas em pesquisas contemporâneas, como, por exemplo, a gestão dos diferentes papéis do atleta na sociedade ${ }^{(55)}$, o tempo despendido na prática deliberada ${ }^{(13)}$, a ordem de fratria ${ }^{(21)}$, ou o efeito relativo da idade ${ }^{(51)}$. No entanto, o reconhecimento destas características, à semelhança do que acontece com outros fatores do treino, não deve ser encarada como uma tentativa de estabelecer um perfil psicossocial rígido passível de fundamentar a identificação de talento. Na realidade, por um lado, a prossecução e manutenção do nível de elite distingue-se por uma diversidade de trajetos possíveis ${ }^{(12)}$ e, por outro, o percurso dos aspirantes a atletas de elite caracteriza-se não apenas pelas etapas clássicas, mas igualmente por micro-transições menos previsíveis ${ }^{\left({ }^{34}\right)}$. Consequentemente torna-se fulcral seguir uma abordagem dialética que integre os processos de identificação, seleção e desenvolvimento, no sentido de explorar novas linhas de investigação que permitam preparar antecipada- 
mente aqueles que ambicionam atingir a excelência desportiva. Efetivamente, durante as respetivas carreiras, estes atletas têm que lidar com transições normativas e não normativas com o intuito de serem bem sucedidas no desporto. Estes períodos sensíveis poderão, em última instância, originar crises nos atletas, caso estes não possuem antecipadamente os recursos de coping necessários ${ }^{(55)}$. Por exemplo, Holt e Mitchell ${ }^{(31)}$ salientam que aspetos como a falta de controlo volitivo, a ausência de planeamento estratégico da respetiva carreira, assim como os deficits ao nível de recursos de coping e suporte tangível poderão dificultar a prossecução de carreiras profissionais em jovens futebolistas com elevado potencial. Partindo destes pressupostos torna-se mais fácil ajudar os jovens jogadores a lidar com as exigências crescentes do desporto de elite.

A presente pesquisa visa comparar diversas características psicológicas de jovens jogadores de elite pertencentes a um clube de topo de futebol em Portugal, divididos em dois grupos em que um é composto por jogadores que se mantiveram no clube em três épocas sucessivas (retidos) e o outro é constituído por praticantes que, no final da primeira ou da segunda época, tiveram que abandonar o clube por decisão do staff técnico (dispensados). A identificação de eventuais diferenças neste domínio permitirá melhorar a compreensão dos atributos requeridos para a transição entre potencial e talento, assim como contribuir para um suporte científico passível de melhorar a preparação de jovens menos bem-sucedidos neste contexto. Com base na revisão de literatura apresentada, estabelecemos como hipótese inicial que os jogadores retidos revelam um perfil psicológico distinto dos seus colegas dispensados.

\section{METODOLOGIA}

\section{PARTICIPANTES}

Centro e trinta e cinco jogadores de futebol integrados num clube de formação de elite português participaram neste estudo. Destes, setenta e dois são jogadores dispensados (não mantiveram a sua participação no clube após o final da primeira ou segunda épocas desportivas) com idades compreendidas entre os treze e os dezoito anos ( $M=15.93$ e DP = .16), e sessenta e três são jogadores retidos (mantiveram a sua participação no clube após o final da primeira ou segunda épocas desportivas) com idades compreendidas entre os catorze e os dezoito anos $(\mathrm{M}=15.26$ e $\mathrm{DP}=.15)$.

\section{INSTRUMENTOS}

Para avaliar a motivação foi utilizada a versão portuguesa ${ }^{(48)}$ da Sport Motivational Scale ${ }^{(42)}$ que inclui 28 itens repartidos por sete subescalas: (i) motivação intrínseca para o conhecimento; (ii) motivação intrínseca para a realização; (iii) motivação intrínseca para a estimulação; (iv) motivação extrínseca identificada; (v) motivação extrínseca introjectada; (vi) motiva- 
ção extrínseca externa; e (vii) amotivação. A resposta ao questionário inclui uma escala Likert de sete pontos que variam entre 1 (não corresponde nada) e 7 (corresponde exatamente).

Quanto ao perfecionismo foi utilizada a versão portuguesa ${ }^{(49)}$ da Multidimensional Perfectionism Scale ${ }^{(15)}$. Este instrumento integra 35 itens distribuídos por seis subescalas: (i) preocupações com os erros; (ii) padrões de realização pessoal; (iii) expectativas parentais; (iv) criticismo parental; (v) dúvidas na ação; e (vi) organização. As respostas a este questionário consideram uma escala tipo Likert de 5 pontos que varia entre 1 (discordo completamente) e 5 (concordo completamente).

Para analisar o suporte parental foi utilizada a versão portuguesa ${ }^{(50)}$ da escala EMBU - Egna Meinnen av Barndoms Uppfostran ${ }^{(43)}$. Este instrumento integra 21 itens distribuídos por três subescalas: (i) suporte emocional; (ii) rejeição; e (iii) superproteção que consideram comportamentos quer por parte dos pais, quer por parte das mães. As respostas as este questionário consideram uma escala tipo Likert de quatro pontos que variam entre 1 (Nunca) e 4 (Sempre).

A resiliência foi avaliada com a versão portuguesa ${ }^{(61)}$ da Resilience Scale ${ }^{(62)}$. Este instrumento integra 25 itens distribuídos por duas subescalas: (i) competência pessoal; e (ii) aceitação do self e da vida. As respostas a este questionário consideram uma escala tipo Likert de 7 pontos que variam entre 1 (concordo totalmente) e 7 (discordo totalmente).

Para estudar as estratégias de confronto utilizou-se a versão portuguesa ${ }^{(41)}$ da escala

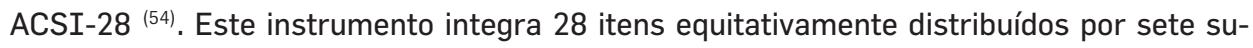
bescalas: (i) rendimento sob pressão; (ii) ausência de preocupações; (iii) confronto com a adversidade; (iv) concentração; (v) formulação de objetivos; (vi) confiança e motivação para a realização; e (vii) treinabilidade. As respostas ao questionário incluem uma escala Likert de 4 pontos, que variam entre 0 (Quase nunca) e 3 (Quase sempre).

A avaliação do compromisso realizou-se com a Escala de Compromisso no Desporto ${ }^{(25)}$. Este instrumento integra 12 itens equitativamente distribuídos por três subescalas: (i) secundarização dos estudos; (ii) isolamento social; e (iii) disciplina de vida. As respostas a este questionário consideram uma escala tipo Likert de 5 pontos que variam entre 1 (discordo totalmente) e 5 (concordo totalmente).

Foi utilizado um questionário sociodemográfico em que se recolheram informações sobre as seguintes variáveis: idade, ano do respetivo grupo de idade ( $1^{\circ}$ ou $2^{\circ}$ ano), trimestre de nascimento, prática de outras modalidades, tempo de prática federada, posição do jogador, número de irmãos, ordem de fratria e residência (internos ou a viver com a família).

\section{PROCEDIMENTOS OPERACIONAIS}

Os questionários foram aplicados em contacto direto com os jogadores após marcação prévia e em sessões dedicadas a esse efeito. As aplicações tiveram lugar numa sala apropriada junto ao local de treino dos participantes. Antes da respetiva aplicação foi agendada 
uma reunião com dirigentes e treinadores responsáveis no sentido de esclarecer os objetivos e procedimentos específicos a considerar na pesquisa, em particular no que concerne ao local e duração prevista para a aplicação dos questionários. Os treinadores garantiram a permissão para a colaboração no estudo e asseguraram o consentimento informado por parte dos atletas. Foi assegurada a confidencialidade dos dados recolhidos e que os mesmos apenas seriam utilizados na investigação em curso. Nos procedimentos foram tidos em conta os princípios éticos para a investigação da Faculdade de Motricidade Humana (Universidade Técnica de Lisboa).

Foi constituída uma base de dados resultante das aplicações dos questionários ao longo de três anos. No final deste período os jogadores sujeitos à avaliação foram divididos em dois grupos de acordo com o critério de se terem mantido nas equipas do clube, ou terem sido dispensados do clube ao cabo do primeiro ou segundo ano.

\section{PROCEDIMENTOS ESTATÍSTICOS}

Os dados foram analisados com o recurso ao software Statistical Package for Social Sciences (v.18.0, PASW Inc., Chicago, IL).

A significância da variável "estatuto no clube" (retido/ dispensado) sobre os diversos construtos psicológicos foi avaliada através da análise de covariância multivariada (MANCOVA), tendo sido definida a variável idade como covariante. Previamente procedeu-se à validação de pressupostos, em particular no que diz respeito à verificação da homogeneidade das matrizes das variancias-covariâncias em cada um dos grupos definidos pelos fatores com o recurso ao teste $M$ de Box. Sempre que foram detetadas evidências de efeitos significativos procederam-se às ANOVAS para cada uma das variáveis dependentes. Foram calculadas as médias, desvios padrão para cada um dos subgrupos e foi considerado um nível de significância de $\alpha=.05$. A classificação da dimensão do efeito foi feita com o $\eta^{2} \mathrm{P}$ de acordo com Maroco ${ }^{(36)}$.

\section{RESULTADOS}

Relativamente às variáveis sociodemográficas, o teste de $M$ Box não revelou diferenças significativas entre as variáveis dependentes (Box's $M=61.02 ; F_{45,56009.349}=1.25, p=.115$ ) e a subsequente MANOVA identificou um efeito multivariado estastisticamente significativo (Pillai's Trace $=0.180, \mathrm{~F}_{9,125}=3.05, \mathrm{p}=.0002, \eta^{2} \mathrm{P}=.180$, potência $=.967$ ). As ANOVAS univariadas confirmaram que os jogadores retidos são mais novos $\left(F_{1,133}=7.72, p=.006, \eta^{2} \mathrm{P}=\right.$ .055) e jogam predominantemente no segundo ano do respetivo grupo de idade $\left(F_{1,133}=8.15\right.$, $\left.p=.005, \eta^{2} \mathrm{P}=.058\right)$. Não foram encontradas evidências de efeitos significativos nas restantes variáveis sociodemográficas, nomeadamente: trimestre de nascimento $\left(\mathrm{F}_{1,133}=.757, \mathrm{p}\right.$ $\left.=.386, \eta^{2} \mathrm{P}=.006\right) ;$ prática de outras modalidades $\left(\mathrm{F}_{1,133}=.143, \mathrm{p}=.706, \eta^{2} \mathrm{P}=.001\right)$; tempo 
dispendido na prática federada $\left(\mathrm{F}_{1,133}=1.29, \mathrm{p}=.257, \eta^{2} \mathrm{P}=.010\right)$; posição em campo $\left(\mathrm{F}_{1,133}\right.$ $\left.=.107, \mathrm{p}=.744, \eta^{2} \mathrm{P}=.001\right)$; número de irmãos $\left(\mathrm{F}_{1,133}=1.45, \mathrm{p}=.230, \eta^{2} \mathrm{P}=.011\right)$; ordem de fratria $\left(F_{1,133}=.708, p=.402, \eta^{2} P=.005\right)$; e residência $\left(F_{1,133}=.485, p=.488, \eta^{2} P=.004\right)$.

No que concerne às variáveis psicossociais, depois de salvaguardada a assunção de homogeneidade das variâncias-covariâncias (Box's $M=721.79 ; F_{496,51574.09}=1.09, p=.072$ ), a MANCOVA evidenciou que o efeito observado do estatuto sobre as variáveis dependentes é significativo (Pillai's Trace $=.383, \mathrm{~F}_{31,102}=2.047, \mathrm{p}=.004, \eta^{2} \mathrm{P}=.383$ ), sendo a potência do teste muito boa (potência $=.996$ ). A análise prosseguiu com os testes $F$ para as respetivas variáveis dependentes (Quadro 1).

QUADRO 1 - Médias, desvios padrão e níveis de significância da MANCOVA.

\begin{tabular}{|c|c|c|c|c|c|c|c|c|}
\hline \multirow[b]{2}{*}{ DIMENSÃO } & \multirow{2}{*}{$\begin{array}{l}\text { NÍVEL DE } \\
\text { PRESTAÇÃO }\end{array}$} & \multirow[b]{2}{*}{$\mathrm{N}$} & \multirow[b]{2}{*}{ M } & \multirow[b]{2}{*}{ DP } & \multicolumn{4}{|c|}{ entre sujeitos } \\
\hline & & & & & $\mathrm{F}$ & $P$ & $\eta^{2} P$ & $\begin{array}{l}\text { Potência } \\
\text { observada }\end{array}$ \\
\hline Secundarização dos & Dispensados & 72 & 2.75 & 1.01 & \multirow{2}{*}{7.42} & \multirow{2}{*}{$.007^{*}$} & \multirow{2}{*}{.053} & \multirow{2}{*}{.772} \\
\hline estudos & Retidos & 63 & 3.11 & 1.16 & & & & \\
\hline Isolamento social & $\begin{array}{l}\text { Dispensados } \\
\text { Retidos }\end{array}$ & $\begin{array}{l}72 \\
63\end{array}$ & $\begin{array}{l}3.84 \\
4.01\end{array}$ & $\begin{array}{l}0.84 \\
0.87\end{array}$ & 3.36 & .069 & .035 & .425 \\
\hline Estilo de vida & $\begin{array}{l}\text { Dispensados } \\
\text { Retidos }\end{array}$ & $\begin{array}{l}72 \\
63\end{array}$ & $\begin{array}{l}4.23 \\
4.43\end{array}$ & $\begin{array}{l}0.60 \\
0.56\end{array}$ & 3.20 & .076 & .024 & .427 \\
\hline $\begin{array}{l}\text { Preocupação com os } \\
\text { erros }\end{array}$ & $\begin{array}{l}\text { Dispensados } \\
\text { Retidos }\end{array}$ & $\begin{array}{l}72 \\
63\end{array}$ & $\begin{array}{l}2.75 \\
2,56\end{array}$ & $\begin{array}{l}0.63 \\
0.77\end{array}$ & .954 & .330 & .007 & .163 \\
\hline Dúvidas sobre a ação & $\begin{array}{l}\text { Dispensados } \\
\text { Retidos }\end{array}$ & $\begin{array}{l}72 \\
63\end{array}$ & $\begin{array}{l}2.81 \\
2.60\end{array}$ & $\begin{array}{l}0.61 \\
0.68\end{array}$ & 4.33 & $.039^{*}$ & .032 & .542 \\
\hline Expectativas parentais & $\begin{array}{l}\text { Dispensados } \\
\text { Retidos }\end{array}$ & $\begin{array}{l}72 \\
63\end{array}$ & $\begin{array}{l}2.44 \\
2.29\end{array}$ & $\begin{array}{l}0.74 \\
0.93\end{array}$ & 2.58 & .111 & .019 & .358 \\
\hline Criticismo parental & $\begin{array}{l}\text { Dispensados } \\
\text { Retidos }\end{array}$ & $\begin{array}{l}72 \\
63\end{array}$ & $\begin{array}{l}1.78 \\
1.59\end{array}$ & $\begin{array}{l}0.52 \\
0.56\end{array}$ & 6.48 & $.012^{*}$ & .047 & .715 \\
\hline $\begin{array}{l}\text { Padrões de realização } \\
\text { pessoal }\end{array}$ & $\begin{array}{l}\text { Dispensados } \\
\text { Retidos }\end{array}$ & $\begin{array}{l}72 \\
63\end{array}$ & $\begin{array}{l}3.68 \\
3.78\end{array}$ & $\begin{array}{l}0.58 \\
0.52\end{array}$ & .233 & .630 & .002 & .077 \\
\hline Organização & $\begin{array}{l}\text { Dispensados } \\
\text { Retidos }\end{array}$ & $\begin{array}{l}72 \\
63\end{array}$ & $\begin{array}{l}4.04 \\
4.08\end{array}$ & $\begin{array}{l}0.54 \\
0.58\end{array}$ & .280 & .597 & .002 & .082 \\
\hline
\end{tabular}




\begin{tabular}{|c|c|c|c|c|c|c|c|c|}
\hline \multirow[b]{2}{*}{ DIMENSÃO } & \multirow{2}{*}{$\begin{array}{l}\text { NÍVEL DE } \\
\text { PRESTAÇÃO }\end{array}$} & \multirow[b]{2}{*}{$\mathrm{N}$} & \multirow[b]{2}{*}{ M } & \multirow[b]{2}{*}{ DP } & \multicolumn{4}{|c|}{ entre sujeitos } \\
\hline & & & & & $\mathrm{F}$ & $\mathrm{P}$ & $\eta^{2} P$ & $\begin{array}{l}\text { Potência } \\
\text { observada }\end{array}$ \\
\hline \multirow{3}{*}{$\begin{array}{l}\text { Motivação intrínseca } \\
\text { (conhecimento) }\end{array}$} & Dispensados & 72 & 5.43 & & \multirow{2}{*}{3.82} & \multirow{2}{*}{$.053^{*}$} & \multirow{2}{*}{.028} & \multirow{2}{*}{.492} \\
\hline & Retidos & 63 & 5.85 & 1.04 & & & & \\
\hline & Dispensados & 72 & 5.44 & 1.29 & \multirow[b]{2}{*}{2.27} & \multirow[b]{2}{*}{.134} & \multirow[b]{2}{*}{.017} & \multirow[b]{2}{*}{.322} \\
\hline $\begin{array}{l}\text { Motivação intrínseca } \\
\text { (realizacão) }\end{array}$ & Retidos & 63 & 5.82 & 1.02 & & & & \\
\hline Motivação intrínseca & $\begin{array}{l}\text { Dispensados } \\
\text { Retidos }\end{array}$ & $\begin{array}{l}72 \\
63\end{array}$ & $\begin{array}{l}5.78 \\
6.30\end{array}$ & $\begin{array}{l}1.07 \\
0.85\end{array}$ & 8.41 & $.004^{*}$ & .060 & .821 \\
\hline $\begin{array}{l}\text { Motivação extrínseca } \\
\text { (identificada) }\end{array}$ & $\begin{array}{l}\text { Dispensados } \\
\text { Retidos }\end{array}$ & $\begin{array}{l}72 \\
63 \\
\end{array}$ & $\begin{array}{l}4.26 \\
3.80 \\
\end{array}$ & $\begin{array}{l}1.46 \\
1.60 \\
\end{array}$ & 3.80 & $.053^{*}$ & .028 & .491 \\
\hline $\begin{array}{l}\text { Motivação extrínseca } \\
\text { (introjectada) }\end{array}$ & $\begin{array}{l}\text { Dispensados } \\
\text { Retidos }\end{array}$ & $\begin{array}{l}72 \\
63\end{array}$ & $\begin{array}{l}4.83 \\
4.51 \\
\end{array}$ & $\begin{array}{l}1.50 \\
1.63 \\
\end{array}$ & 1.48 & .176 & .014 & .271 \\
\hline $\begin{array}{l}\text { Motivação extrínseca } \\
\text { (externa) }\end{array}$ & $\begin{array}{l}\text { Dispensados } \\
\text { Retidos }\end{array}$ & $\begin{array}{l}72 \\
63 \\
\end{array}$ & $\begin{array}{l}3.95 \\
3.50 \\
\end{array}$ & $\begin{array}{l}1.32 \\
1.61\end{array}$ & 5.48 & $.021^{*}$ & .040 & .643 \\
\hline Amotivação & $\begin{array}{l}\text { Dispensados } \\
\text { Retidos }\end{array}$ & $\begin{array}{l}72 \\
63\end{array}$ & $\begin{array}{l}1.78 \\
1.40\end{array}$ & $\begin{array}{l}1.10 \\
0.74\end{array}$ & 8.03 & $.005^{*}$ & .057 & .803 \\
\hline $\begin{array}{l}\text { Resiliência - } \\
\text { Competência }\end{array}$ & $\begin{array}{l}\text { Dispensados } \\
\text { Retidos }\end{array}$ & $\begin{array}{l}72 \\
63\end{array}$ & $\begin{array}{l}5.58 \\
5.91\end{array}$ & $\begin{array}{l}0.66 \\
0.65\end{array}$ & 10.12 & $.002^{*}$ & .071 & .885 \\
\hline Resiliencia - Aceitação & $\begin{array}{l}\text { Dispensados } \\
\text { Retidos }\end{array}$ & $\begin{array}{l}72 \\
63 \\
\end{array}$ & $\begin{array}{l}5.07 \\
5.27 \\
\end{array}$ & $\begin{array}{l}0.58 \\
0.76\end{array}$ & 2.41 & .122 & .018 & .339 \\
\hline Rendimento sob pressão & $\begin{array}{l}\text { Dispensados } \\
\text { Retidos }\end{array}$ & $\begin{array}{l}72 \\
63\end{array}$ & $\begin{array}{l}2.15 \\
2.17\end{array}$ & $\begin{array}{l}0.64 \\
0.59\end{array}$ & 1.28 & .250 & .010 & .203 \\
\hline $\begin{array}{l}\text { Ausência de } \\
\text { preocupações }\end{array}$ & $\begin{array}{l}\text { Dispensados } \\
\text { Retidos }\end{array}$ & $\begin{array}{l}72 \\
63\end{array}$ & $\begin{array}{l}1.81 \\
1.92\end{array}$ & $\begin{array}{l}0.69 \\
0.70\end{array}$ & .711 & .400 & .005 & .133 \\
\hline $\begin{array}{l}\text { Confronto com a } \\
\text { adversidade }\end{array}$ & $\begin{array}{l}\text { Dispensados } \\
\text { Retidos }\end{array}$ & $\begin{array}{l}72 \\
63\end{array}$ & $\begin{array}{l}1.64 \\
1.73 \\
\end{array}$ & $\begin{array}{l}0.59 \\
0.60 \\
\end{array}$ & .619 & .433 & .005 & .122 \\
\hline Concentração & $\begin{array}{l}\text { Dispensados } \\
\text { Retidos }\end{array}$ & $\begin{array}{l}72 \\
63\end{array}$ & $\begin{array}{l}1.89 \\
1.85\end{array}$ & $\begin{array}{l}0.53 \\
0.51\end{array}$ & 0.27 & .830 & .000 & .053 \\
\hline Definição de objetivos & $\begin{array}{l}\text { Dispensados } \\
\text { Retidos }\end{array}$ & $\begin{array}{l}72 \\
63\end{array}$ & $\begin{array}{l}1.72 \\
1.83\end{array}$ & $\begin{array}{l}0.72 \\
0.68\end{array}$ & .468 & .495 & .004 & .104 \\
\hline $\begin{array}{l}\text { Confiança e motivação } \\
\text { para realização }\end{array}$ & $\begin{array}{l}\text { Dispensados } \\
\text { Retidos }\end{array}$ & $\begin{array}{l}72 \\
63\end{array}$ & $\begin{array}{l}2.19 \\
2.28\end{array}$ & $\begin{array}{l}0.51 \\
0.46\end{array}$ & .351 & .555 & .003 & .090 \\
\hline Treinabilidade & $\begin{array}{l}\text { Dispensados } \\
\text { Retidos }\end{array}$ & $\begin{array}{l}72 \\
63\end{array}$ & $\begin{array}{l}2.30 \\
2.31\end{array}$ & $\begin{array}{l}0.43 \\
0.48\end{array}$ & .044 & .835 & .000 & .055 \\
\hline
\end{tabular}




\begin{tabular}{|c|c|c|c|c|c|c|c|c|}
\hline \multirow{2}{*}{ DIMENSÃO } & \multirow{2}{*}{$\begin{array}{l}\text { NÍVEL DE } \\
\text { PRESTAÇÃO }\end{array}$} & \multirow{2}{*}{$\mathrm{N}$} & \multirow{2}{*}{$M$} & \multirow{2}{*}{ DP } & \multicolumn{4}{|c|}{ entre sujeitos } \\
\hline & & & & & $\mathrm{F}$ & $\mathrm{P}$ & $\eta^{2} P$ & $\begin{array}{l}\text { Potência } \\
\text { observada }\end{array}$ \\
\hline \multirow{3}{*}{ Rejeição do pai } & Dispensados & 72 & 1.19 & 0.27 & \multirow{2}{*}{3.02} & \multirow{2}{*}{.084} & \multirow{2}{*}{.022} & \multirow{2}{*}{.408} \\
\hline & Retidos & 63 & 1.17 & 0.26 & & & & \\
\hline & Dispensados & 72 & 1.22 & 0.29 & \multirow{2}{*}{4.23} & \multirow{2}{*}{$.042^{*}$} & \multirow{2}{*}{.031} & \multirow{2}{*}{.086} \\
\hline Rejeição da mãe & Retidos & 63 & 1.16 & 0.33 & & & & \\
\hline \multirow{2}{*}{ Superproteção do pai } & Dispensados & 72 & 2.29 & 0.49 & \multirow{2}{*}{.975} & \multirow{2}{*}{.325} & \multirow{2}{*}{.007} & \multirow{2}{*}{.165} \\
\hline & Retidos & 63 & 2.26 & 0.49 & & & & \\
\hline \multirow{2}{*}{ Superproteção da mãe } & Dispensados & 72 & 2.50 & 0.49 & \multirow{2}{*}{1.64} & \multirow{2}{*}{.203} & \multirow{2}{*}{.012} & \multirow{2}{*}{.246} \\
\hline & Retidos & 63 & 2.43 & 0.50 & & & & \\
\hline \multirow[t]{2}{*}{ Suporte do pai } & Dispensados & 72 & 3.46 & 0.47 & \multirow{2}{*}{.315} & \multirow{2}{*}{.575} & \multirow{2}{*}{.002} & \multirow{2}{*}{.086} \\
\hline & Retidos & 63 & 3.51 & 0.57 & & & & \\
\hline \multirow[t]{2}{*}{ Suporte da mãe } & Dispensados & 72 & 3.63 & 0.31 & \multirow{2}{*}{.007} & \multirow{2}{*}{.933} & \multirow{2}{*}{.000} & \multirow{2}{*}{.051} \\
\hline & Retidos & 63 & 3.63 & 0.44 & & & & \\
\hline
\end{tabular}

Nota: ${ }^{*} \leq .05$

Os resultados identificaram diferenças significativas nas seguintes dimensões: secundarização dos estudos $\left(\mathrm{F}_{1,133}=7.42, \mathrm{p}=.007, \eta^{2} \mathrm{P}=.053\right)$; dúvidas sobre a ação $\left(\mathrm{F}_{1,133}=\right.$ 4.33, $\left.\mathrm{p}=.039, \eta^{2} \mathrm{P}=.032\right)$; criticismo parental $\left(\mathrm{F}_{1,133}=6.48, \mathrm{p}=.012, \eta^{2} \mathrm{P}=.047\right)$; motivação intrínseca - conhecimento $\left(\mathrm{F}_{1,133}=3.82, \mathrm{p}=.053, \eta^{2} \mathrm{P}=.028\right)$; motivação intrínseca - estimulação $\left(\mathrm{F}_{1,133}=8.41, \mathrm{p}=.004, \eta^{2} \mathrm{P}=.060\right)$; motivação extrínseca identificada $\left(\mathrm{F}_{1,133}=\right.$ 3.80, $\left.\mathrm{p}=.053, \eta^{2} \mathrm{P}=.028\right)$; motivação extrínseca externa $\left(\mathrm{F}_{1,133}=5.48, \mathrm{p}=.021, \eta^{2} \mathrm{P}=.040\right)$; amotivação $\left(F_{1,133}=8.03, p=.005, \eta^{2} \mathrm{P}=.057\right)$; resiliência - competência $\left(F_{1,133}=10.12, p=\right.$ $\left..002, \eta^{2} \mathrm{P}=.071\right)$; e rejeição da mãe $\left(\mathrm{F}_{1,133}=4.23, \mathrm{p}=.042, \eta^{2} \mathrm{P}=.031\right)$.

\section{DISCUSSÃo}

No que diz respeito às variáveis sociodemográficas, os jogadores retidos são mais novos e jogam predominantemente no segundo ano do respetivo grupo de idade. Estas diferenças derivam da gestão dos recursos humanos do clube uma vez que o número de jogadores que constituem os plantéis dos diferentes escalões vai diminuindo à medida que os jogadores transitam para os grupos de idade mais avançados (e.g., 65 jogadores nos sub 15, 45 jogadores nos sub 17 e 25 jogadores nos sub 19). Consequentemente, em cada época desportiva, um maior número de jogadores transita do escalão sub 15 para o escalão sub 
17 comparativamente com a transição seguinte em que estão apenas disponíveis sensivelmente 12 vagas (transição de sub 17 para sub 19). Esta redução de jogadores entre escalões torna também mais acessível a transição de praticantes mais novos no sentido de se manterem no clube entre o primeiro e o segundo ano do mesmo grupo de idade (e.g., sub 14 para sub 15), comparativamente com os colegas que têm que lidar com a transição entre escalões (e.g., sub 15 para sub 17).

No que concerne às variáveis psicossociais, os jogadores retidos demonstraram um compromisso mais elevado com o desporto, estando mais disponíveis para demorar mais tempo a finalizar os estudos, para faltar às aulas ou até para desistir dos seus projetos académicos no sentido de reforçar a sua participação desportiva. Apesar de estes aspetos não constituírem uma condição para serem bem-sucedidos no plano desportivo, em particular nas fases iniciais de formação, a gestão dos domínios desportivo e académico torna-se mais difícil quando se equaciona a transição para o futebol profissional. Na realidade, são bem conhecidas as dificuldades dos jovens jogadores de futebol de elite em conciliar as carreiras desportiva e académica com sucesso ${ }^{(6)}$. Efetivamente, após a etapa de especialização desportiva, as prestações de nível de elite requerem a disponibilidade para despender milhares de horas na prática deliberada. Consequentemente, alguns atletas de elite assumem o desporto como uma prioridade nas suas vidas reconhecendo que, para aí serem bem-sucedidos, alguns sacrifícios têm que ser feitos ${ }^{(45)}$. Considerando o contexto específico do futebol, parece consensual que para atingir os mais elevados níveis de prestação, esta determinação para fazer sacrifícios é crucial ${ }^{(30,31)}$ e em consequência é razoável considerar o compromisso como um dos construtos psicológicos mais relevantes neste domínio. Esta capacidade de empenho e de operacionalização de comportamentos autoimpostos tem particular relevância para os atletas que não se consideram particularmente promissores nas etapas iniciais de participação desportiva ${ }^{(34)}$.

Os jogadores retidos revelaram também valores significativamente mais baixos nas dimensões dúvidas sobre a ação e criticismo parental, aspeto que parece ser vantajoso na medida em que os torna mais próximos de um perfil de perfecionistas ajustados. $\mathrm{Na}$ realidade, de acordo com Hamachek ${ }^{(26)}$, elevados níveis de realização pessoal podem surgir associados quer ao perfecionismo neurótico, quer ao perfecionismo ajustado. Tendo em consideração a escala de perfecionismo de Frost et al. ${ }^{(15)}$ utilizada nesta pesquisa, o perfecionismo ajustado concilia elevados níveis de organização e padrões de realização pessoal com baixas pontuações nas restantes dimensões negativas da escala, nomeadamente, a preocupação com os erros, as dúvidas sobre a ação, o criticismo parental e as expectativas parentais. Este perfil mais ajustado (ou saudável) parece surgir associado a indivíduos mais bem-sucedidos nos domínios académico ${ }^{(27)}$ e desportivo ${ }^{(23)}$. Por outro lado, apesar dos perfecionistas desajustados poderem expressar, igualmente, elevados níveis de organização e padrões de realização pessoal, estes surgem associados a pontuações 
altas nas dimensões negativas do perfecionismo anteriormente mencionadas. Esta última conjugação surge na literatura associada a fenómenos como a orientação para o ego ${ }^{(10)}$, cólera ${ }^{(58)}$, esgotamento ${ }^{(24)}$, baixa autoestima ${ }^{(22)}$ ou diminuição da qualidade das prestações perante o feedback negativo ${ }^{(3)}$. Considerando as diferenças identificadas ao nível do criticismo parental, esta pesquisa reforça a relevância do papel desempenhado pelos pais neste domínio uma vez que, aparentemente, as orientações motivacionais dos pais parecem moldar as tendências perfecionistas dos filhos. De facto, existem evidências de que os filhos tendem a sentir-se pressionados para serem bem-sucedidos quando os seus pais expressam elevados níveis de expectativas e criticismo parental ${ }^{(2)}$.

Os jogadores retidos revelam igualmente um perfil mais autodeterminado corroborando a teoria da autodeterminação ${ }^{(9)}$ e o modelo da motivação intrínseca e extrínseca ${ }^{(59)}$, pois, aparentemente, quanto mais autodeterminados os comportamentos dos praticantes, melhores são as prestações desportivas ${ }^{(18)}$. Por outro lado, os perfis menos autodeterminados tendem a surgir associados às piores prestações ${ }^{(19)}$. No entanto, estes últimos autores também constataram que atletas de alto rendimento conciliam elevados níveis de motivação autónoma e controlada, reforçando as conclusões de Mallet e Hanrahan ${ }^{(35)}$ quando referem que as recompensas externas têm um impacto relevante na perceção de competência dos atletas de elite contribuindo para a sua autodeterminação. Outra explicação plausível para os valores elevados ao nível da motivação externa poderá estar relacionada com a aspiração dos atletas a tornarem-se famosos. Neste caso, os elevados níveis de motivação autónoma poderão ter uma função protetora em contraposição com a motivação controlada ${ }^{(19)}$. Considerando os valores, moderados, ao nível da motivação extrínseca detetados no presente estudo, podemos colocar igualmente a hipótese da regulação externa aumentar ao longo do tempo até que os atletas atinjam o nível sénior, onde aparentemente as recompensas externas assumem maior relevância.

Os jogadores retidos exibem, ainda, níveis mais elevados de resiliência. Vários aspetos poderão suportar estas diferenças, reforçando a perspetiva multidimensional deste construto, nomeadamente no que concerne aos recursos pessoais, estratégias de coping ou influências socioculturais ${ }^{(17)}$. Consequentemente, para além dos níveis mais elevados de autodisciplina e determinação, os jogadores retidos demonstram maior controlo emocional requerido para atingir os seus objetivos, o que parece ser um recurso determinante no contexto do futebol. Na realidade, jovens jogadores de futebol bem-sucedidos no seu trajeto até ao nível sénior profissional demonstram maior persistência na prossecução dos seus objetivos ao longo do tempo ${ }^{(64)}$, em particular quando assumem a responsabilidade pessoal pelas suas rotinas de treino numa perspetiva volitiva ${ }^{(31)}$. Este importante fator reforça as requeridas competências autoregulatórias que permitem aos atletas demonstrar maior autonomia e responsabilidade pelo seu processo de desenvolvimento a longo prazo, em vez de estarem simplesmente orientados para corresponder às exigências dos 
treinadores. Segundo Toering, Elferink-Gemser, Jordet e Visscher ${ }^{(57)}$, sem estas competências os jogadores tendem a gerir de forma inadaptada os seus insucessos. Podemos, igualmente, salientar o estatuto dos jogadores, uma vez que os retidos são mais bem-sucedidos, contando-se entre estes alguns atletas internacionais. Este aspeto pode assumir particular relevância na medida em que estão numa fase crítica de desenvolvimento da sua identidade e autoavaliam-se como jogadores excecionais capazes de prestações acessíveis apenas a uma minoria de praticantes ${ }^{(56)}$. De facto, o reconhecimento por parte dos treinadores das suas capacidades, o qual se reflete nas convocatórias para as competições, proporciona um importante suporte, o que por sua vez tem impacto favorável no desenvolvimento da resiliência (7).

Ao nível do suporte parental, mais uma vez, os jogadores retidos parecem ganhar vantagem teórica neste domínio, uma vez que demonstram valores significativamente mais baixos ao nível da rejeição da mãe. Estes resultados estão de acordo com as expetativas, considerando a existência de uma associação entre as atitudes parentais de rejeição e superproteção, e os elevados níveis de traço e de estado de ansiedade ${ }^{(14)}$, desajustamentos na fase inicial da adolescência, ${ }^{(47)}$, sintomas psicopatológicos ${ }^{(39)}$ ou baixos níveis de autoconceito ${ }^{(40)}$. Por outro lado, é bem conhecido o papel dos pais no processo de desenvolvimento de talento em particular no que concerne ao suporte emocional e tangível frequentemente relatados pelos atletas de elite ${ }^{(4,8)}$, assim como a associação entre o sucesso na carreira desportiva e a propensão para solicitar suporte social no sentido de lidar com as adversidades ${ }^{(64)}$.

Considerando, ainda, o facto de que normalmente a motivação dos jovens para o contexto académico é menos autodeterminada comparativamente com o contexto desportivo (5), na presente amostra é possível que os pais dos jogadores dispensados, com base nos resultados visíveis, questionem mais os seus filhos quanto ao seu investimento no desporto. Neste sentido, poderão levantar mais dúvidas acerca das prioridades estabelecidas por estes, estimulando mais frequentemente a sua reflexão sobre as suas expetativas futuras. Tal posição, mais crítica, poderá frenar o investimento destes jogadores numa eventual carreira como futebolistas profissionais ao admitirem terem menos probabilidades realistas de se manterem no clube em que estão integrados.

\section{CONCLUSÕES}

Os jogadores retidos demonstram um perfil psicossociológico mais ajustado, em particular no que concerne a uma perceção de suporte parental mais elevada, a um perfecionismo mais ajustado, assim como a níveis de compromisso, de resiliência e de autodeterminação mais elevados. Estes resultados reforçam a literatura existente sobre os processos de 
desenvolvimento de talento e manutenção dos níveis de elite, que apontam para a relevância de tais características. Como referido anteriormente, os intervenientes no sistema desportivo deverão considerar que estas características não deverão suportar procedimentos de seleção dicotómicos, mas, em alternativa, contribuir para o fomento de características técnicas, táticas, físicas e psicossociais deficitárias no âmbito de um processo multidimensional e longitudinal. Justifica-se assim a reflexão sobre possíveis modelos de desenvolvimento que encarem este fenómeno numa ótica mais personalizada correspondendo, desta forma, à diversidade de contextos e fatores (assim como as inúmeras combinações entre os mesmos) suscetíveis de contribuir para a prossecução da excelência desportiva. Adicionalmente, é importante ponderar o conceito de talento num contexto bem mais abrangente, que considere a diversidade de estímulos, sobretudo nas etapas iniciais de aprendizagem. De facto, a desejada transição entre potencial e talento depende da qualidade e da diversidade de estímulos passíveis de fomentar nas crianças e nos jovens áreas de interesse e de maior apetência. Ambientes com tais características, em particular em contexto escolar, contribuem para o desenvolvimento de um autoconceito em áreas específicas de competência (e.g. artes, ciências, desporto, etc.), assim como o respetivo reconhecimento social, que em conjunto constituem importantes alicerces neste âmbito.

Este estudo encerra ainda algumas limitações sobre as quais importa refletir. Em primeiro lugar porque centra a atenção apenas nas percepções dos jogadores, não integrando assim informações provenientes de outros intervenientes, em particular dos pais e dos treinadores dos atletas. Por outro lado, recorre a uma abordagem transversal, o que impossibilita a análise da evolução dos resultados dos jogadores retidos e dispensados ao longo do tempo, na sequência das opções tomadas pelos treinadores.

Futuras pesquisas deverão pois ponderar a conciliação de metodologias qualitativas e quantitativas numa perspetiva multidimensional e longitudinal, e que considere os diversos intervenientes no processo de desenvolvimento do talento. 
1. Abbott A, Collins D (2004). Eliminating the dichotomy between theory and practice in talent identification and development: considering the role of psychology. Journal of Sport Sciences, 22: 395-408.

2. Ablard KE, Parker WD (1997). Parents' Achievement Goals and Perfectionism in their academically talented children. Journal of Youth and Adolescence, 26 (6): 651-667.

3. Anshel MH, Mansouri H (2005). Influences of perfectionism on motor performance, affect, and causal attributions in response to critical information feedback. Journal of Sport Behavior 28 (2): 99-124.

4. Bloom BS (1985). Generalizations about talent development. In: Bloom BS (ed), Developing talent in young people. New York: Ballantine Books, 507-549.

5. Bois J, Sarrazin P (2007). Self-Determination of Contextual Motivation, Inter-Context Dynamics and Adolescents' Patterns of Sport Participation over Time. Psychology of Sport and Exercise, 8: 685-703.

6. Christensen MK, Sørensen JK (2009): Sport or school? Dreams and dilemmas for talented young Danish football players. European Physical Education Review, 15 (1): 115-133.

7. Connaughton D, Hanton S, Jones G (2010). The Development and Maintenance of Mental Toughness in the World's Best Performers. The Sport Psychologist, 24: 168-193.

8. Côté J (1999). The influence of the family in the development of talent in sport. The Sport Psychologist, 13: 395-417.

9. Deci EL, Ryan RM (1985). Intrinsic motivation and self-determination in human behavior. New York: Plenum.

10. Dunn JGH, Dunn JC, Syrotuik DG (2002). Relationship Between Multidimensional Perfectionism and Goal Orientations in Sport. Journal of Sport \& Exercise Psychology, 24: 376-395.

11. Durand-Bush N, Salmela JH (2001). The development of talent in sport. In: Singer RN, Hausenblas HA, Janelle CM (eds.), Handbook of sport psychology (2nd ed). New York: John Wiley \& Sons, Inc, 269-289.
12. Durand-Bush N, Salmela JH (2002). The development and maintenance of expert athletic performance: Perceptions of World and Olympic champions. Journal of Applied Sport Psychology, 14: 154-171.

13. Ericsson KA, Krampe R, Tesch-Romer C (1993). The role of deliberate practice in the acquisition of expert performance. Psychological Review, 199: 363-406.

14. Festa CC, Ginsburg GS (2011). Parental and Peer Predictors of Social Anxiety in Youth. Child Psychiatry Human Development, 42: 291-306.

15. Frost RO, Marten P, Lahart CM, Rosenblate R (1990). The dimensions of perfectionism. Cognitive Therapy and Research, 14: 449-468.

16. Gagné $F$ (2009). Building gifts into talents: Detailed overview of the DMGT 2.0. In: MacFarlane B, Stambaugh $\mathrm{T}$ (eds.), Leading change in gifted education: The festschrift of Dr. Joyce VanTassel-Baska. Waco, TX: Prufrock Press, 61-80.

17. Galli N, Vealey RS (2008). "Bouncing Back" From Adversity: Athletes' Experiences of Resilience. Sport Psychologist, 22: 316-335.

18. Gillet N, Berjot S, Gobancé L (2009): A motivational model of performance in the sport domain, European Journal of Sport Science, 9 (3): 151-158.

19. Gillet N, Vallerand RJ, Rosnet E (2009). Motivational clusters and performance in a real-life setting. Motivation \& Emotion, 33: 49-62.

20. Golby J, Sheard M (2004). Mental toughness and hardiness at different levels of rugby league. Personality and Individual Differences, 37: 933-942.

21. Gottfried AW, Gottfried AE, Bathurst K, Guerin DW (1994). Gifted IQ: Early developmental aspects. The Fullterton longitudinal study. New York: Plenum.

22. Gotwals JK, Dunn JG, Wayment HA (2003). An examination of perfectionism and self-esteem in intercollegiate athletes. Journal of Sport Behavior, 26 (1): 18-38.

23. Gould D, Dieffenbach K, Moffett A (2002). Psychological characteristics and their development in Olympic champions. Journal of Applied Sport Psychology, 14: 172-204. 
24. Gould D, Guinan D, Greenleaf C, Medbery R, Peterson K (1999). Factors affecting Olympic performance: perceptions of athletes and coaches from more and less successful teams. The Sport Psychologist, 13: 371-394.

25. Gouveia EF, Serpa S (2006). Escala de compromisso no desporto. Documento não puvlicado, Universidade Técnica de Lisboa.

26. Hamachek DE (1978). Psychodynamics of normal and neurotic perfectionism. Psychology: A Journal of Human Behavior, 15: 27-33.

27. Hanchon TA (2010). The relations between perfectionism and achievement goals. Personality and Individual Differences, 49: 885-890.

28. Helsen WF, Hodges NJ, Winckel JV, Starkes JL (2000). The roles of talent, physical precocity and practice in the development of soccer expertise. Journal of Sport Sciences, 18: 727-736.

29. Helsen WF, Winckel JV, Williams AM (2005). 'The relative age effect in youth soccer across Europe. Journal of Sports Sciences, 23 (6): 629 - 636.

30. Holt NL, Dunn JG (2004). Toward a grounded theory of the psychosocial competencies and environmental conditions associated with soccer success. Journal of Applied Sport Psychology, 16: 199-219.

31. Holt NL, Mitchell T (2006). Talent development in English professional soccer. International Journal of Sport Psychology, 37: 77-98.

32. Jonker L, Elferink-Gemser MT, Toering TT, Lyons J, Visscher C (2010). Academ ic performance and self-regulatory skills in elite youth soccer players. Journal of Sports Sciences, 28 (14): 1605-1614.

33. Lesma ML, Pérez-González B, Salinero JJ (2011). Relative age effect (RAE) in Spanish football league. Journal of Sport and Health Research. 3 (1) : 35-46.

34. MacNamara A, Button A, Collins D (2010). The Role of Psychological Characteristics in Facilitating the Pathway to Elite Performance Part 2: Examining Environmental and Stage-Related Differences in Skills and Behaviors. The Sport Psychologist, 24: 74-96.
35. Mallett CJ, Hanrahan SJ (2004). Elite athletes: Why does the "fire" burn so brightly? Psychology of Sport and Exercise, 5: 183-200.

36. Maroco JP (2010) Análise Estatística com o PASW Statistics (ex-SPSS). ReportNumber: Pêro Pinheiro.

37. Martindale RJJ, Collins D, Daubney J (2005). Talent development: A guide for practice and research within sport. Quest, 57: 353-375.

38. Morris T (2000). Psychological characteristics and talent identification in soccer. Journal of Sport Sciences, 18: 715-726.

39. Muris P, Meesters C, Van Brakel A (2003). Assessment of Anxious Rearing Behaviors with a Modified Version of "Egna Minnen Betr"affande Uppfostran" Questionnaire for Children. Journal of Psychopathology and Behavioral Assessment, 25 (4): 229-338.

40. Nishikawa S, Sundbom E, Hägglöf B (2010). Influence of Perceived Parental Rearing on Adolescent Self-Concept and Internalizing and Externalizing Problems in Japan. Journal of Child and Family Studies: 19, 57-66.

41. Palmeira A, Serpa S (1997). ACSI28 - Estudo das experiências desportivas (versão portuguesa). Lisboa: Faculdade de Motricidade Humana - Universidade Técnica de Lisboa.

42. Pelletier LG, Fortier MS, Vallerand RJ, Tuson KM, Brière NM, Blais MR (1995). Toward a new measure of intrinsic motivation, extrinsic motivation, and amotivation in sports: The Sport Motivational Scale (SMS). Journal of Sport \& Exercise Psychology, 17: 35-53.

43. Perris $C$, Jacobson L, Lindstörm $H$, Von Knorring L, Perris H (1980). Development of a new inventory for assessing memories of parental rearing behaviour. Ata Psychiatrica Scandinavica, 61: 265-274.

44. Phillips E, Keith D, Renshaw I, Portus M (2010). Expert performance in sport and the dynamics of talent development. Sports Medicine, 40 (4) : 271-283.

45. Scanlan TK, Russell DG, Beals KP, Scanlan LA (2003). Project on elite athlete commitment (PEAK): II. A direct test and expansion of the sport commit- 
ment model with elite amateur sportsmen. Journal of Sport \& Exercise Psychology, 25: 377-401.

46. Scanlan TK, Russell DG, Magyar TM Scanlan LA (2009). Project on elite athlete commitment (PEAK): III. An Examination of the external validity across gender, and the expansion and clarification of the sport commitment model. Journal of Sport \& Exercise Psychology, 31: 685-705.

47. Sentse M, Veenstra R, Lindenberg S, Verhulst FC, Ormel $J$ (2009). Buffers and risks in temperament and family for early adolescent psychopathology: Generic, conditional, or domain specific effects? The TRAILS study. Developmental Psychology, 45: 419-430.

48. Serpa S, Alves P, Barreiros A (2004a). Versão portuguesa da Sport Motivational Scale (SMSp): processos de tradução, adaptação e fiabilidade. Lisboa: Laboratório de Psicologia do Desporto - Faculdade de Motricidade Humana - Universidade Técnica de Lisboa. 49. Serpa S, Alves P, Barreiros A. (2004b). Versão portuguesa da Multidimensional Perfectionism Scale (MPSp): processos de tradução, adaptação e fiabilidade. Lisboa: Laboratório de Psicologia do Desporto - Faculdade de Motricidade Humana Universidade Técnica de Lisboa.

50. Serpa S, Alves P, Barreiros A (2004c). Versão portuguesa do Egna Minnen av Barndoms Uppfostran (EMBUp): processos de tradução, adaptação e fiabilidade. Lisboa: Laboratório de Psicologia do Desporto - Faculdade de Motricidade Humana Universidade Técnica de Lisboa.

51. Sherar LB, Baxter-Jones ADG, Faulkner RA, Russell KW (2007). Do physical maturity and birth date predict talent in male youth ice hockey players? Journal of Sports Sciences, 25 (8): 879 - 886.

52. Simmons C, Paull GC (2001) 'Season-of-birth bias in association football. Journal of Sports Sciences, 19 (9): 677 - 686.

53. Simonton DK (1999). Talent and its development: an emergenic and a epigenetic model. Psychological Review, 106 (3): 435-457.

54. Smith RE, Schutz RW, Smoll FL, Ptacek JT (1995). Development and validation of a multidimensional measure of sport-specific psychological skills: The Athletic Coping Skills Inventory-28. Journal of Sport and Exercise Psychology, 17 (4): 379-398.

55. Stambulova N (2009). Talent development in sport: A career transitions perspetive. In: Tsung-Min Hung E, Lidor R, Hackfort D (eds.) Psychology of Sport Excellence. Morgantown, WV: Fitness Information Technology, 63-74.

56. Stephan Y, Brewer BW (2007). Perceived determinants of identification with the athlete role among elite competitors. Journal of Applied Sport Psychology, 19 (1): 67-79.

57. Toering TT, Elferink-Gemser MT, Jordet G, Visscher C (2009). Self-regulation and performance level of elite and non-elite youth soccer players. Journal of Sports Sciences, 27 (14): 1509-1517.

58. Vallance JKH, Dunn JGH, Dunn JLC (2006). Perfectionism, Anger, and Situation Criticality in Competitive Youth Ice Hockey. Journal of Sport \& Exercise Psychology, 28: 383-406.

59. Vallerand RJ (1997). Toward a hierarchical model of intrinsic and extrinsic motivation. In: Zanna MP (ed.), Advances in experimental social psychology New York: Academic Press, 271-360.

60. Vaeyens R, Philippaerts RM, Malina RM (2005). The relative age effect in soccer: $A$ match-related perspetive. Journal of Sports Sciences, 23 (7): 747 - 756.

61. Vigário I, Serpa S (2007). Versão portuguesa da Resilience Scale (RSp): processos de tradução, adaptação e fiabilidade. Lisboa: Laboratório de Psicologia do Desporto - Faculdade de Motricidade Humana - Universidade Técnica de Lisboa.

62. Wagnild G, Young HM (1993) Development and psychometric evaluation of the Resilience Scale. Journal of Nursing Measurement, 1: 165-177.

63. Williams AM, Reilly T (2000). Talent identification and development in soccer. Journal of Sport Sciences, 18: 657-667.

64. Yperen NV (2009). Why some make it and others do not: Identifying psychological factors that predict career success in professional adult soccer. The Sport Psychologist, 23: 317-329. 